ISBN: 978-980-427-021-5

\title{
El fenómeno del fútbol como herramienta para la formación integral en la primera infancia
}

\author{
Andrés Leonardo Colorado Arango ${ }^{1}$
}

\section{Resumen}

La presente investigación tuvo como finalidad el realizar un análisis de las capacidades físicas de los niños con 5 años cumplidos que hacen parte del programa de iniciación deportiva en primera infancia denominado "Cantera de Héroes" del Envigado F.C., con la meta de determinar la incidencia de los procesos desarrollados en cada una de las sesiones y actividades en el desarrollo físico-motriz y en la calidad de vida de los participantes que como caso particular son acompañados durante el desarrollo de los entrenamientos por sus padres o acudientes. Para la valoración de las variables de las capacidades físicas se utilizó una batería de test físicos validados para niños entre los 3 y 6 años por (Ayan P., 2013), obteniendo resultados muy alentadores sobre la propuesta del Envigado F.C., ya que, uno de los principales hallazgos de la investigación demostró que la variable que generó mayor diferencia en los resultados fue la del año de ingreso al programa, confirmándose que los niños con mayor tiempo en la actividad presentaron consistentemente mejores resultados que los niños que ingresaron el presente año al proyecto, por lo cual, se confirma la pertinencia del programa de iniciación deportiva en primera infancia denominado "Cantera de Héroes" del Envigado F.C. para el desarrollo integral de niños en la primera infancia con un gran aporte al mejoramiento de la calidad de vida.

Palabras clave: desarrollo motriz, calidad de vida, primera infancia.

1 Universidad de San Buenaventura - Medellín, Colombia. Correo electrónico: andreslc24@gmail.com, Licenciado en Educación Física y Deportes. Magíster en Administración, Campo de Conocimiento: Iniciación Deportiva y Gestión de Programas, Grupo de Investigación: Estudios Interdisciplinarios en Educación (ESINED). Línea: Educación Física, Cuerpo y Motricidad. 


\section{INTRODUCCIÓN}

La calidad de vida debe entenderse como uno de los principales objetivos que se proyectan los diferentes grupos sociales, ya que, la evolución en los sistemas agrícolas, productivos, de salud, de transporte, etc., buscan aportar en cierta medida al confort y el aumento de la expectativa de vida de los individuos, entendiendo que un ser humano sano, será más productivo.

El sector de la educación física y el deporte ha generado propuestas que tienen como objetivo el aportar al mejoramiento de las condiciones de vida de sus usuarios, pero el lograr evidenciar el verdadero impacto de manera empírica ha sido complicado por la poca producción investigativa en este campo, además de las controversias que se generan en los círculos académicos frente a afirmaciones como "el deporte es salud", cuando se observan atletas que por la exigencia competitiva cargan con dolores crónicos, lesiones y desgastes óseos y musculares que les limita al final de sus carreras hasta las acciones más sencillas y cotidianas.

En este aspecto, el Envigado Fútbol Club como equipo profesional miembro de la división mayor del fútbol colombiano se ha caracterizado a lo largo de su historia por su interés en formar deportistas con proyección nacional e internacional, hecho que lo ha llevado a recibir el título de "las mejores divisiones inferiores del país" por la cantidad de futbolistas con una importante trayectoria en el ámbito local y trasnacional que han surgido de la institución.

Este reconocimiento ha sido refrendado por los excelentes resultados obtenidos por el club en eventos futbolísticos de categorías menores como el pony fútbol y los torneos organizados por la liga antioqueña de fútbol, además de participaciones en eventos nacionales e internacionales a nivel de clubes, la convocatoria de sus jugadores para selecciones Antioquia y el gran aporte a los seleccionados nacionales en categorías sub 15 y sub 17.

En el año 2014 después del excelente campeonato mundial que realizó la selección nacional de Colombia en Brasil donde obtuvo el 5to. puesto como el papel estelar de varios jugadores surgidos del Envigado F.C., la demanda de servicios de enseñanza del fútbol cada vez a más temprana edad, llevó a los directivos de la organización a tomar la decisión de implementar un proyecto de iniciación deportiva para niños entre 2 y 6 años que sirva como plataforma para el desarrollo de las habilidades motrices básicas y las capacidades condicionales de los niños para lograr ventajas al momento de iniciar la fundamentación técnica y la preparación física específica para 
el fútbol, sin dejar de lado que el aporte esencial del programa debe ser el aporte al desarrollo integral de los niños y el mejoramiento de la calidad de vida a través del deporte y que el alto rendimiento debe darse como consecuencia de un proceso de iniciación y formación deportiva pertinente.

En el contexto colombiano se encuentra que los programas de iniciación deportiva a temprana edad y específicamente en el fútbol, se han desarrollado basados en la demanda de padres de familia que buscan formar jugadores profesionales desde la primera etapa de la vida de sus hijos, con una mentalidad de hacer cualquier cosa para lograr que su hijo pueda cumplir los sueños frustrados de sus progenitores, pero lo más delicado es que muchas organizaciones asumen el proceso de iniciar tempranamente a niños en un deporte específico sólo por la rentabilidad económica que puede entregar el proceso sin tener en cuenta el impacto físico y psicológico que genera un programa deportivo en el desarrollo de un infante y las repercusiones a largo plazo que pueden traer.

Al realizar un rastreo de la bibliografía científica en el tema de iniciación deportiva, no se encuentran muchos trabajos que realicen un seguimiento a las variables físico-motrices, además es insuficiente la información que se comparte a los padres de familia sobre la evolución de sus hijos en el proceso, lo que genera desmotivación y abandono del deporte.

El campo de la iniciación deportiva en la primera infancia en Colombia, aún es incipiente frente a producción científica que sirva como referencia para la construcción de propuestas pertinentes para la población entre 0 y 6 años y teniendo en cuenta que en el medio existe una gran oferta en programas deportivos que tienen como usuarios a esta población, es necesario que la academia se preocupe por realizar un proceso investigativo que permita evidenciar el comportamiento de las variables que se trabajan en el proyecto.

El objetivo de la investigación se centró en realizar una valoración de las capacidades físicas en los niños de 5 años cumplidos que hacen parte del proyecto de iniciación deportiva en la primera infancia denominado "cantera de Héroes" del Envigado F.C. por medio de la batería de test propuestas por (Ayan P., 2013) en los siguientes ámbitos:

- Para la medición de la resistencia aeróbica se utilizó la prueba "3 minutes shuttle run test" para niños entre 4 y 5 años validada por (Oja \& Juerimaee, 1997) con un valor $r=0.74-0.80$, la cual tiene como objetivo medir en metros la distancia recorrida por un sujeto en tres minutos. 
- Para determinar la Fuerza Explosiva en miembro inferior se aplicó la prueba "standing long jump test" para niños entre 3 y 6 años, validada por Bos, et all (2004) con un valor $r>0.80$, la cual tiene como objetivo medir en metros la distancia que logra un sujeto al saltar de un punto fijo hacia adelante con ambos pies.

- La agilidad se estimó con la prueba " 10 x 5 m shuttle run test" para niños de 5 años validada por Fjørtft (2011) con un valor $r=0.8$, la cual busca determinar en segundos y décimas de segundo el tiempo que demora un sujeto en completar 10 recorridos de 5 metros cambiando de orientación.

- Finalmente, para medir la velocidad se aplicó una modificación de la prueba "25 meter dash" para niños entre 4 y 6 años, validada por Ikeda \& Aoyagi (2008) con un valor $r=0.73$, la cual busca determinar en segundos y décimas de segundo el tiempo que demora un sujeto en completar un recorrido lineal para este caso de 20 metros por la dimensión del terreno de juego en el que se desarrollan los entrenamientos del programa.

De igual forma se tomaron medidas antropométricas como el peso corporal, la estatura y el índice de masa corporal para correlacionarlos con valores obtenidos en las pruebas.

Los datos fueron tomados en campo utilizando implementos de medición cómo cintas métricas, básculas, tallímetros y cronómetros con precisión garantizada por los fabricantes, estos a su vez fueron ingresados en planillas físicas y luego alojados en una matriz en Microsoft Excel versión 2010.

Finalmente es importante resaltar que sólo hicieron parte de la población de análisis los niños que asistieron mínimamente al 80\% de los entrenamientos programados por la coordinación del proyecto y fueron autorizados por sus padres de familia por escrito para hacer parte de la muestra con el compromiso por parte de los investigadores de guardar absoluta reserva con los datos de identificación familiar y la seguridad que los datos obtenidos sólo se utilizarán con fines académicos.

\section{MARCO TEÓRICO Y REVISIÓN DE LITERATURA}

El desarrollo de un programa de iniciación deportiva a temprana edad ha sido controvertido por los teóricos como (Orts D., 2008) debido a que las estructuras corporales de los niños y niñas aún no se encuentran lo suficientemente maduras para soportar impactos y cargas de entrenamiento, inclusive, llegando a afectar el normal crecimiento óseo y muscular, además de las tensiones que se generan entorno a la competencia entre padres de 
familia y entrenadores, ya que, en muchas situaciones la comunicación y el acompañamiento no son los mejores

Ilustración 1. Niños del Proyecto Cantera de Héroes

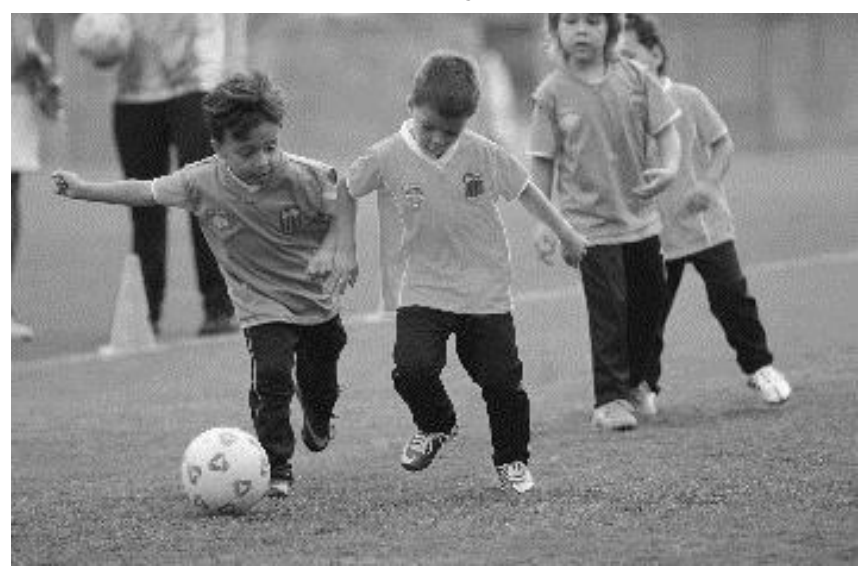

Otro de los factores que genera reserva en los círculos académicos son los procesos atencionales de los niños y las necesidades afectivas, ya que, el iniciar un proceso deportivo requiere de algunas demandas de nivel sensorial y de pensamiento elaborado, además de un nivel de autonomía que le permita tomar decisiones e introyectar conceptos técnicos y tácticos, que los infantes en su proceso de desarrollo cognitivo y socio afectivo aún no han alcanzado.

Ilustración 2. Participación padres de Familia en las sesiones de Entrenamiento

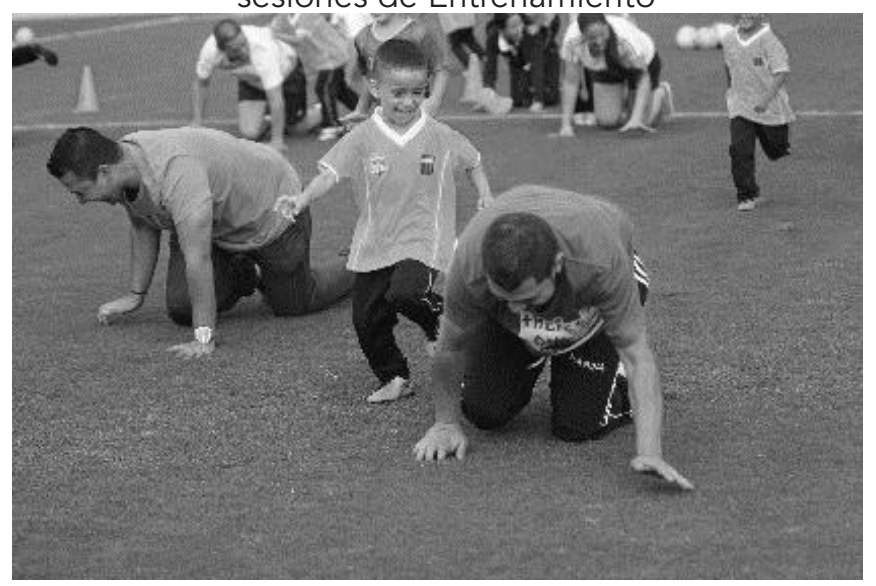

La gran controversia se centra en los planteamientos de teóricos como (Tabernero S., Marquez, \& Llanos, 2002) donde se exponen factores como la falta de formación de los orientadores de los programas, la escasa bibliografía y producción investigativa en el tema y los factores ambientales que afectan el proceso como los padres de familia, el contexto sociocultural y los escenarios e implementos deportivos destinados para el proceso, lo que ha conducido a una especie de ambigüedad e indeterminación frente a la edad óptima para la iniciación en programas deportivos, además que en la oferta de servicios de actividad física en los primeros años de vida, las promesas de valor o los objetivos varían dependiendo de la organización que los oferte y el perfil 
del personal que los orienta.

Ilustración 3. Programa Orientado por Profesionales del área de la Educación Física y el Deporte

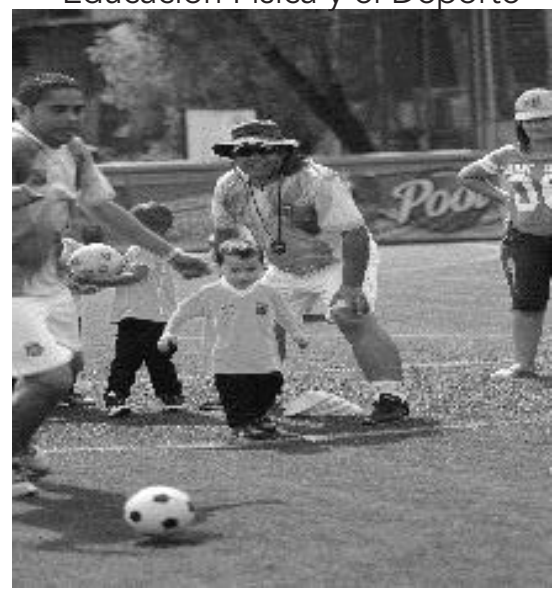

El fenómeno del fútbol a nivel mundial ha llegado a generar un deseo por la especialización temprana de niños con el objetivo de formar jugadores cada vez más fuertes, veloces, resistentes, inteligentes y efectivos, por lo cual, se asumen posturas de planificación de sesiones deportivas para niños en primera infancia que no tienen en cuenta su desarrollo corporal y emocional, generando un estrés inmediato que desencadenará inevitablemente la deserción. (Pérez C. \& Llames L., 2010).

La iniciación al fútbol se configura como un ejercicio de aprendizaje deportivo específica en una modalidad concreta, por tanto, es recomendable que en etapas anteriores se realice una estimulación motriz multilateral y otra de fase de familiarización con la lógica interna de los deportes colectivos de invasión, coincidiendo con autores como (López R. \& Castejón O., 2005) Esto no quiere decir que no sea aconsejable enseñar fútbol antes de los 12 años, como lo hace el Envigado F.C., se plantea que en las edades tempranas debe enfatizarse como la motricidad general y otros deportes tratados de forma inespecífica y sólo iniciar el proceso de formación técnico específico una vez se asegure un excelente acervo motor.

De acuerdo con lo anterior el programa que ofrece Cantera de Héroes utiliza el fútbol como una herramienta para el desarrollo motriz del infante, planteando todas sus actividades desde referentes teóricos de gran autoridad en el ámbito del desarrollo en la primera infancia como es Arnold Gessel, una autoridad histórica y de talla mundial que conceptuó frente a las etapas del desarrollo en los primeros años de vida, como resultado de numerosas observaciones y extensas investigaciones sobre el desarrollo motriz, el desarrollo del lenguaje y el ámbito psicosocial para lograr una propuesta pertinente y beneficiosa. (Weizmann \& Harris, 2012)

A nivel mundial se han realizado estudios experimentales en niños con edades entre los 3 y los 6 años que participan programas deportivos en como el realizado por (Jiménez D. \& Araya V., 2009) donde se analiza el efecto de una intervención motriz en el desarrollo motor, el rendimien- 
to académico y la creatividad en niños preescolares concluyendo sobre las ventajas de los niños que desarrollan actividades deportivas sobre los que no las desarrollan en las variables estudiadas, otra investigación realizada por (López S., Borrego B., \& Díaz S., 2013) demuestra como los efectos de un programa de actividad física inciden en la composición corporal de niños entre 3 y 5 años, dejando claro que dichos programas generan una mejora significativa sobre la masa grasa.

Otro de los grandes referentes teóricos para este trabajo investigativo es la propuesta formulada por (Ayan P., 2013) donde se realiza una revisión diferentes baterías de test que han sido validadas para la medición de las variables físico motrices que se trabajan en los programas deportivos en el Contexto de la Educación Infantil, es fundamental resaltar que estos protocolos han sido aplicados en el contexto europeo.

\section{EL CASO CANTERA DE HÉROES DEL ENVIGADO F.C.}

El proyecto de iniciación deportiva en primera infancia denominado "Cantera de Héores" del Envigado F.C. tiene como objetivo el contribuir al desarrollo integral de los niños a través de un proceso de estructuración motriz a partir de los fundamentos básicos de la práctica del fútbol.

El proyecto pretende que los niños alcancen su máximo potencial motriz y fortalezcan el vinculo con sus padres utilizando el fenómeno del fútbol como un elemento que genera una gran motivación para grandes y chicos.

Una de las estrategias de la propuesta del Envigado F.C. es que los niños aprendan jugando atraves de la imitacion, ya que, la primera infancia es una etapa de aprendizaje motor sensible; de ahí la importancia del acompañamiento de los padres en cada una de las clases.

Este plan emprendido por el Envigado FC a partir del año 2014 atiende niños desde los 2 hasta los 7 años y pretende que el niño se divierta y aprenda jugando, ademas se fortalecen valores como el respeto, la tolerancia el acatamiento de la norma, apuntando a una verdadera formacion holistica del ser humano y se hace importante en la medida que este acompañado por los padres de familia, ya que esta es la base fundamental de la sociedad.

Las clases están acompañadas por dos docentes y grupos máximo de 15 niños, permitiendo que el proceso tenga un mejor desarrollo; La filosofía del Envigado FC se centra en el trabajo de calidad con personal altamente calificado, instalaciones deportivas en las mejores condiciones e implementación deportiva pertinente. 
La aceptación que ha tenido el proyecto por parte de la academia, los padres de familia y el sector deporte en general, lleva a pensar en un plan de expansión de esta metodología para contribuir con el óptimo desarrollo motriz de los niños del área metropolitana del Valle de Aburrá y de esta forma aportar a la formación integral, el fortalecimiento de los vínculos padres e hijos y a la consolidación de hábitos de vida saludables en la población infantil antioqueña.

\section{ANÁLISIS DE RESULTADOS}

Las pruebas físicas se aplicaron a 43 niños que cumplieron con los criterios de selección de la muestra y que además fueron autorizados por sus padres para participar de la investigación en los días 13, 20 y 27 de febrero del año 2016 en canchas de grama sintética ubicadas en el estadio polideportivo sur de envigado.

La aplicación de las pruebas físicas fue ejecutada por 4 auxiliares de investigación estudiantes de la tecnología en entrenamiento deportivo de la universidad de San Buenaventura Medellín, los cuales fueron capacitados tanto en la teoría como en la práctica para llevar a cabo dicha actividad.

Una vez realizada la tabulación de los resultados para los 43 niños, se efectuó el análisis estadístico del mismo, con los valores numéricos correspondientes y según las unidades estipuladas para la valoración de cada prueba:

- Prueba de Velocidad en 20 metros: Segundos y décimas de segundo

- Prueba de Agilidad: Segundos y décimas de segundo

- Prueba de Fuerza Explosiva: Centímetros

- Prueba de Resistencia: Metros

Igualmente se definieron las medidas estadísticas que se utilizaron para analizar la información:

- El Promedio como la media aritmética y se calcula sumando un grupo de números y dividiéndolo por el recuento de dichos números

- Moda es el número que aparece con mayor frecuencia dentro de una muestra

- La desviación estándar como una medida de dispersión para variables cuantitativas o cantidades racionales y de intervalo. Se conoce por la raíz cuadrada de la varianza y mide cuando se separan los datos.

- El coeficiente de variación como un dato que expresa la desviación estándar como porcentaje, facilitando el análisis del grado de va- 
riabilidad en los datos. Este consiste en la relación entre la desviación estándar y el promedio. Para la muestra se considera que una estimación con un coeficiente de variación:

- 0 a $20 \%$ es excelente

- 21 a $40 \%$ es aceptable

- 41 en adelante, promedio no representativo para la población.

Los datos se tabularon y procesaron utilizando el programa Microsoft Excel 2010 del cual se extrajeron los datos estadísticos y los gráficos para el análisis.

Para el análisis de los resultados se realizó una agrupación por edades para facilitar la interpretación y las conclusiones.

Perfil antropométrico de los niños del proyecto cantera de héroes del envigado F.C.

Como se mencionó anteriormente la investigación realizó un análisis de las capacidades físicas en niños con 5 años cumplidos que hacen parte del proyecto de iniciación deportiva en primera infancia denominado Cantera de Héroes del Envigado F.C., pero antes de pasar al análisis de los resultados en cada una de las pruebas es importante definir los patrones antropométricos de los niños evaluados.

En el ámbito del peso corporal, se encuentra que el grupo de niños evaluados tiene un peso promedio de 17.8 kilogramos con una desviación estándar de 2.51 kilogramos y un coeficiente de variación de sólo el 14\%, evidenciando que no existen grandes diferencias en esta variable, por lo cual, el desempeño en las pruebas físicas no se verá afectado positiva o negativamente por el peso corporal.

En el tema de la estatura, las diferencias son mucho menores, ya que, la talla promedio fue de 1.07 metros, con una desviación estándar de 3 centímetros y un coeficiente de evaluación de sólo el 3\%, por lo cual, también se puede determinar que los resultados en las pruebas no tendrán sesgos por la estatura corporal.

Como relación entre el peso y la estatura se encuentra que el Índice de Masa Corporal para los niños evaluados se encuentra en promedio 15.43 kilogramos sobre metros cuadrados, una desviación estándar de 1.76 kilogramos sobre metros cuadrados y como consecuencia un coeficiente de variación del $11 \%$.

Finalmente, se observó la proporción de niños evaluados por año de ingreso al programa, observando que el 53\% ingresó en el año 2015, el 35\% en el año 2016 y sólo el 12\% viene en el proyecto desde el año 2014 cuando 
se iniciación las actividades (ilustración 1)

Ilustración 4. Distribución por año de ingreso de los niños evaluados

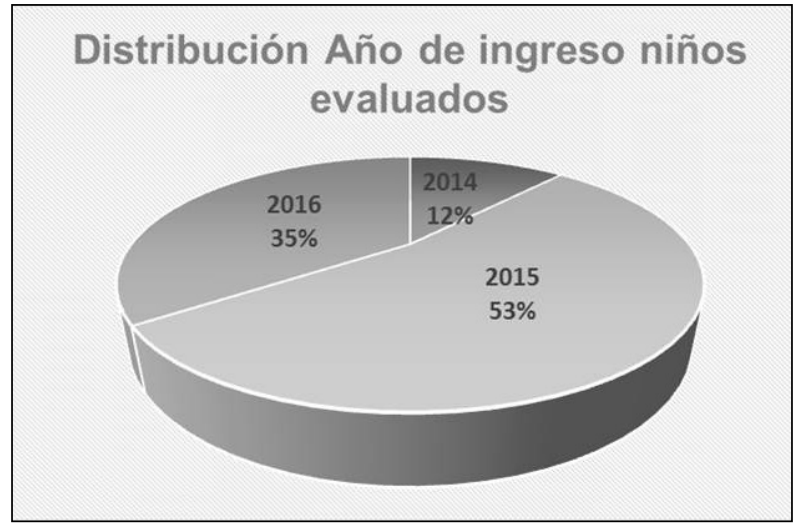

\section{Resultados pruebas de valoración física}

\section{Prueba de velocidad}

La prueba para determinar la velocidad máxima se desarrolló según el siguiente protocolo:

Posición inicial de pie, se debe correr lo más rápido posible a lo largo de una superficie recta de 30 metros de longitud. Se crono- metra el tiempo que se tarda en recorrer los primeros 25 metros. Es aconsejable realizar la prueba por parejas. (Ayan P., 2013: 60)

Para el caso de esta investigación se realizó una modificación a la distancia a cronometrar, ya que, el espacio en el que se desarrollan los entrenamientos tenía una longitud de sólo 28 metros, por lo cual, se decidió cronometrar sólo los primeros 20 metros de recorrido para evitar cualquier tipo de accidente.

La prueba se desarrolló invitando al padre de familia a correr delante del niño para generar una motivación al tratar de alcanzarlo y de esta manera acercarse a la velocidad máxima real que pueda alcanzar el niño.

La Tabla 1 presenta los resultados generales para la prueba de velocidad, destacándose un tiempo promedio de 6.23 segundos y un coeficiente de variación del $13 \%$ que entrega una buena confiabilidad a dicha media, ya que, el rango entre el valor mínimo (4.64 segundos) y el valor máximo (7.46 segundos) fue de sólo 2.82 segundos: 
Tabla 1. Resumen resultados de la prueba de velocidad en 20 metros.

\begin{tabular}{|c|c|}
\hline Estadístico & Valor Numérico \\
\hline Promedio & 6,23 \\
\hline D. Estándar & 0,79 \\
\hline C. Variación & $13 \%$ \\
\hline Moda & S/D \\
\hline Mínimo & 4,64 \\
\hline Máximo & 7,46 \\
\hline Rango & 2,82 \\
\hline
\end{tabular}

Fuente: Elaboración propia

Para el análisis se realizó un filtro sobre el año de ingreso al programa, buscando determinar diferencias en los resultados que puedan estar relacionados con la cantidad de tiempo que lleva el niño participando de las actividades y se encontraron los datos relacionados en la tabla 2:

Tabla 2. Resumen resultados de la prueba de velocidad de 20 metros diferenciados por año de ingreso al programa.

\begin{tabular}{|c|c|c|c|}
\hline Estadístico & Resultado 2014 & Resultado 2015 & Resultado 2016 \\
\hline Promedio & 5,45 & 6,28 & 6,33 \\
\hline D. Estándar & 0,51 & 0,78 & 0,84 \\
\hline C. Variación & $9 \%$ & $12 \%$ & $13 \%$ \\
\hline Moda & S/D & S/D & S/D \\
\hline Mínimo & 5,09 & 4,64 & 5,27 \\
\hline Máximo & 5,81 & 7,46 & 7,43 \\
\hline Rango & 0,72 & 2,82 & 2,16 \\
\hline
\end{tabular}

Fuente: Elaboración propia

Luego de comprobar que los promedios obtenidos representan excelentemente los datos resumidos en él según el coeficiente de variación que para los tres grupos de datos fue inferior al $20 \%$ se pasó a realizar una comparación del rendimiento en la prueba para los tres grupos, observándose un mejor rendimiento promedio en los niños más antiguos en el programa (ilustración 5), además al analizar los resultados de las pruebas utilizando el coeficiente de Pearson se encuentra una correlación directa baja (0.20), indicando la tendencia que a mayor antigüedad en el programa el tiempo en la prueba fue menor. 
Ilustración 5. Comparativo resultados prueba de velocidad por año de ingreso al programa.

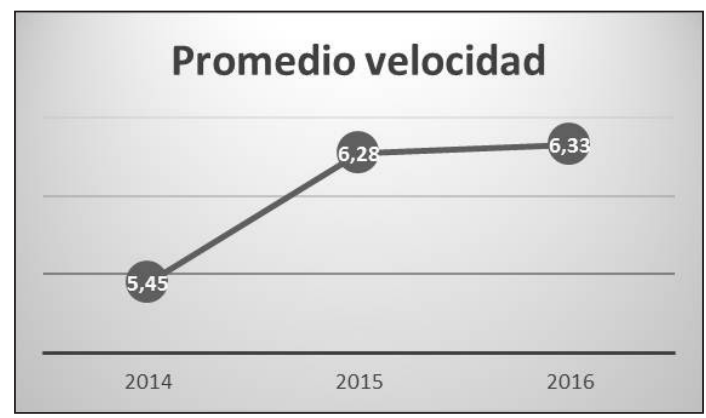

Fuente: Elaboración Propia

\section{Prueba de Agilidad}

La prueba para determinar la agilidad se desarrolló según el siguiente protocolo:

Se dibujan dos líneas rectas ( $1 \mathrm{~m}$ aprox. de longitud) separadas $5 \mathrm{~m}$ entre sí. Partiendo de una de las mismas, se debe llegar a la otra con ambos pies y regresar al punto de partida (un ciclo). Se cronometra el tiempo que necesario para completar cinco ciclos. Ante cualquier error en la ejecución, la prueba se detiene y debe ser repetida. (Ayan P., 2013: 59)

La Tabla 3 presenta los resultados generales para la prueba de agilidad, destacándose un tiempo promedio de 30.14 segundos y un coeficiente de variación del $11 \%$ que entrega una buena confiabilidad a dicha media, aunque llama la atención que el valor máximo está alejado por lo menos 10 segundos del valor de la media, puede inferirse este dato como un outlier o valor atípico que no afecta en gran medida los estadísticos de tendencia central y dispersión:

Tabla 3. Resumen resultados de la prueba de agilidad.

\begin{tabular}{|c|c|}
\hline Estadístico & Valor Numérico \\
\hline Promedio & 30,14 \\
\hline D. Estándar & 3,28 \\
\hline C. Variación & $11 \%$ \\
\hline Moda & 29,87 \\
\hline Mínimo & 24,75 \\
\hline Máximo & 40,09 \\
\hline Rango & 15,34 \\
\hline
\end{tabular}


Fuente: Elaboración propia

Para el análisis se realizó un filtro sobre el año de ingreso al programa, buscando determinar diferencias en los resultados que puedan estar relacionados con la cantidad de tiempo que lleva el niño participando de las actividades y se encontraron los datos relacionados en la tabla 4:

Tabla 4. Resumen resultados de la prueba de agilidad diferenciados por año de ingreso al programa.

\begin{tabular}{|c|c|c|c|}
\hline Estadístico & Resultado 2014 & Resultado 2015 & Resultado 2016 \\
\hline Promedio & 29,68 & 29,79 & 30,80 \\
\hline D. Estándar & 4,65 & 3,68 & 2,55 \\
\hline C. Variación & $16 \%$ & $12 \%$ & $8 \%$ \\
\hline Moda & S/D & S/D & S/D \\
\hline Mínimo & 26,39 & 24,75 & 25,96 \\
\hline Máximo & 32,97 & 40,09 & 34,97 \\
\hline Rango & 6,58 & 15,34 & 9,01 \\
\hline
\end{tabular}

Fuente: Elaboración propia

Luego de comprobar que los promedios obtenidos representan excelentemente los datos resumidos para los tres periodos evaluados, se pasó a realizar una comparación del rendimiento en la prueba para los tres grupos, observándose un mejor rendimiento promedio en los niños más antiguos en el programa (ilustración 6), además al analizar los resultados de las pruebas utilizando el coeficiente de Pearson se encuentra una correlación directa baja (0.13), indicando la tendencia que a mayor antigüedad en el programa el tiempo en la prueba fue menor.

Ilustración 6. Comparativo resultados prueba de agilidad por año de ingreso al programa.

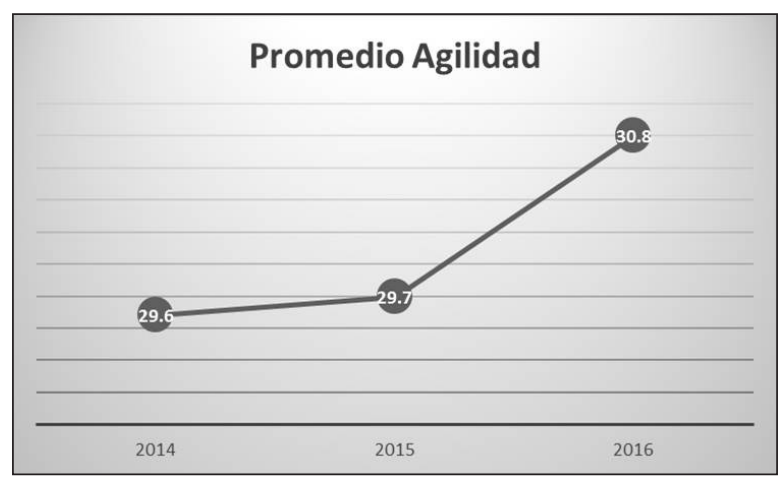

Fuente: Elaboración Propia. 


\section{Prueba de Fuerza Explosiva}

La prueba para determinar la fuerza explosiva en miembros inferiores se desarrolló según el siguiente protocolo:

Salto a dos pies desde una línea recta. Se permite balanceo previo de brazos y flexión de rodillas. Se debe aterrizar con ambos pies sin que las manos toquen el suelo. Se realizan dos intentos, se registra la mayor distancia alcanzada en uno de ellos. (Ayan P., 2013: 59)

La Tabla 5 presenta los resultados generales para la prueba de fuerza explosiva, destacándose un avance promedio en el salto de 92.78 centímetros y un coeficiente de variación del 17\% que entrega una buena confiabilidad a dicha media, aunque llama la atención que el valor mínimo está alejado por lo menos 42 centímetros del valor de la media, puede inferirse este dato como un outlier o valor atípico que no afecta en gran medida los estadísticos de tendencia central y dispersión:

Tabla 5. Resumen resultados de la prueba de fuerza explosiva.

\begin{tabular}{|c|c|}
\hline Estadístico & Valor Numérico \\
\hline Promedio & 92,78 \\
\hline D. Estándar & 15,91 \\
\hline C. Variación & $17 \%$ \\
\hline Moda & 92 \\
\hline Mínimo & 50 \\
\hline Máximo & 115 \\
\hline Rango & 65 \\
\hline
\end{tabular}

Fuente: Elaboración propia

Para el análisis se realizó un filtro sobre el año de ingreso al programa, buscando determinar diferencias en los resultados que puedan estar relacionados con la cantidad de tiempo que lleva el niño participando de las actividades y se encontraron los datos relacionados en la tabla 6.

Tabla 6. Resumen resultados de la prueba de fuerza explosiva diferenciados por año de ingreso al programa.

\begin{tabular}{|c|c|c|c|}
\hline Estadístico & Resultado 2014 & Resultado 2015 & Resultado 2016 \\
\hline Promedio & 92 & 94,94 & 89,40 \\
\hline D. Estándar & 10,2 & 17,27 & 14,59 \\
\hline C. Variación & $10 \%$ & $18 \%$ & $16 \%$ \\
\hline Moda & S/D & 108 & 90 \\
\hline
\end{tabular}




\begin{tabular}{|c|c|c|c|}
\hline Estadístico & Resultado 2014 & Resultado 2015 & Resultado 2016 \\
\hline Mínimo & 82 & 50 & 70 \\
\hline Máximo & 98 & 109 & 115 \\
\hline Rango & 20 & 59 & 45 \\
\hline
\end{tabular}

Fuente: Elaboración propia

Luego de comprobar que los promedios obtenidos representan excelentemente los datos resumidos para los periodos evaluados, se pasó a realizar una comparación del rendimiento en la prueba para los tres grupos, observándose un mejor rendimiento promedio en los niños que ingresaron en el año 2015 por encima de los ingresados 2014, mientras que los que iniciaron el proceso en 2016, obtuvieron en promedio una distancia menor en el salto (ilustración 7), además al analizar los resultados de las pruebas utilizando el coeficiente de Pearson se encuentra una correlación inversa baja (-0.14), indicando la tendencia que a mayor antigüedad en el programa el tiempo en la distancia en el salto fue mayor.

Ilustración 7. Comparativo resultados prueba de fuerza explosiva por año de ingreso al programa.

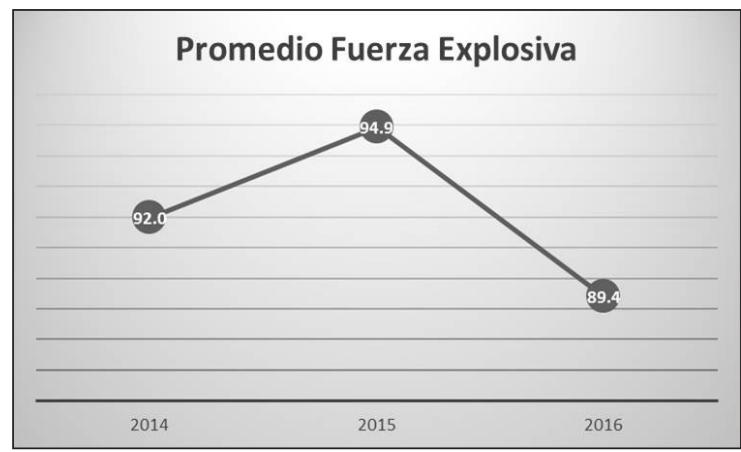

Fuente: Elaboración Propia

\section{Prueba de Resistencia}

La prueba para determinar la resistencia aeróbica se desarrolló según el siguiente protocolo:

Separar 10 m entre sí dos postes, se valora la distancia en metros que el niño/a recorre durante tres minutos realizando sucesivas carreras de ida y vuelta alrededor de los mismos. Para la prueba se demarco un cuadrado de 40 metros con 10 metros de cada lado para que los niños realizaran la carrera continua durante los 3 minutos establecidos y de esta manera se contabilizara de manera más ágil la distancia recorrida. (Ayan P., 2013: 58) 
La Tabla 7 presenta los resultados generales para la prueba de resistencia, destacándose un avance promedio en el recorrido de 346 metros y un coeficiente de variación del $19 \%$ que entrega una buena confiabilidad a dicha media, aunque llama la atención que el valor mínimo está alejado por lo menos 146 metros del valor de la media, puede inferirse este dato como un outlier o valor atípico que no afecta en gran medida los estadísticos de tendencia central y dispersión:

Tabla 7. Resumen resultados de la prueba de resistencia.

\begin{tabular}{|c|c|}
\hline Estadístico & Valor Numérico \\
\hline Promedio & 346,96 \\
\hline D. Estándar & 65,60 \\
\hline C. Variación & $19 \%$ \\
\hline Moda & 380 \\
\hline Mínimo & 200 \\
\hline Máximo & 450 \\
\hline Rango & 250 \\
\hline
\end{tabular}

Fuente: Elaboración propia

Para el análisis se realizó un filtro sobre el año de ingreso al programa, buscando determinar diferencias en los resultados que puedan estar relacionados con la cantidad de tiempo que lleva el niño participando de las actividades y se encontraron los datos relacionados en la tabla 8:

Tabla 8. Resumen resultados de la prueba de resistencia diferenciados por año de ingreso al programa.

\begin{tabular}{|c|c|c|c|}
\hline Estadístico & Resultado 2014 & Resultado 2015 & Resultado 2016 \\
\hline Promedio & 280,00 & 373,08 & 321,25 \\
\hline D. Estándar & 56,57 & 55,28 & 67,28 \\
\hline C. Variación & $20 \%$ & $15 \%$ & $21 \%$ \\
\hline Moda & S/D & 380 & 360 \\
\hline Mínimo & 240 & 250 & 200 \\
\hline Máximo & 320 & 450 & 400 \\
\hline Rango & 80 & 200 & 200 \\
\hline
\end{tabular}

Fuente: Elaboración propia

Luego de comprobar que los promedios obtenidos representan excelentemente los datos resumidos para los periodos evaluados, se pasó a realizar una comparación del rendimiento en la prueba para los tres grupos, 
observándose un mejor rendimiento promedio en los niños que ingresaron en el año 2015 por encima de los ingresados 2016, mientras que los que iniciaron el proceso en 2014, obtuvieron en promedio una distancia menor en el recorrido (ilustración 8), además al analizar los resultados de las pruebas utilizando el coeficiente de Pearson se encuentra una correlación inversa media (-0.42), indicando la tendencia que a mayor antigüedad en el programa el tiempo en la distancia recorrida fue mayor.

llustración 8. Comparativo resultados prueba de resistencia por año de ingreso al programa.

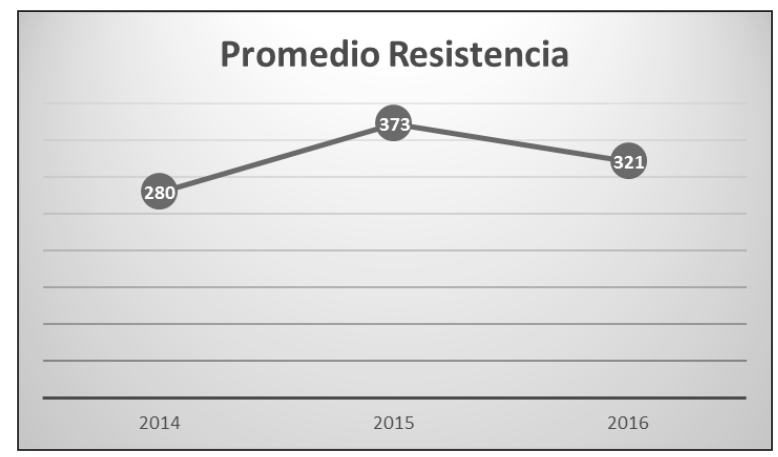

Fuente: Elaboración Propia

\section{CONCLUSIONES}

Luego de observar los resultados se encuentra que el proceso que se ha desarrollado en el programa ha generado impactos verificables en las capacidades físicas de los niños participantes del programa, es más, se puede confirmar que existe una relación entre el tiempo en el programa y los resultados en las pruebas físicas aplicadas, ya que, la evidencia estadística muestra una tendencia de mejores resultados en todas las capacidades para los niños que ingresaron al programa en los años 2014 y 2015 frente a los niños que iniciaron las actividades deportivas en el 2016.

Igualmente es importante resaltar que el grupo de niños evaluado que ingresó en el programa en el año 2015, que configuró el 53\% de la población, fue el que evidenció mejores resultados en las pruebas de fuerza explosiva y resistencia, mientras que en las pruebas de velocidad y agilidad estuvo ligeramente por debajo del grupo de niños que ingresaron en 2014, teniendo en cuenta que el inicio de las actividades deportivas fue sólo hasta el mes de octubre de 2014.

Se destaca el resultado de la correlación entre la cantidad de metros recorridos en la prueba de resistencia y el año de ingreso al programa con 
un coeficiente de Pearson del -0.42 , ya que, esto demuestra una correlación inversa media entre las variables, indicando que los que ingresaron en los años 2014 y 2015 recorrieron en promedio más metros que los niños que ingresaron al programa en 2016 y si hablamos de la resistencia como una capacidad física que depende de la utilización eficiente del oxígeno como fuente de energía y para que para ello requiere el correcto funcionamiento de los sistemas cardiovascular y respiratorio, se puede concluir que el impacto del programa sobre el mejoramiento de la salud física de los niños es evidente.

Los resultados de la investigación muestran que los niños con mayor antigüedad en el programa ostentan ventajas en las capacidades físicas frente a niños de su misma edad, similar peso corporal y semejante estatura que dejan ver una contribución empírica del programa Cantera de Héroes del Envigado F.C. al desarrollo integral de los niños participantes y por ende aportando al mejoramiento de la calidad de vida como consecuencia de un proceso estructurado y orientado por profesionales del área de la educación física y el deporte que utilizan el fútbol como una herramienta motivadora y transformadora que impacta las dinámicas de las familias que participan de las actividades semana a semana y que se ven beneficiados al encontrar un espacio que posibilita el sano esparcimiento, la unión familiar, el fortalecimiento de las relaciones entre padres e hijos, el encuentro de sensaciones gratificantes que desencadenan hábitos de vida saludable que acompañarán a estos niños durante todas sus vidas, ya que, el desarrollo de la personalidad y los principales rasgos que configuran las relaciones interpersonales se aprenden en este periodo de la vida.

El proceso de iniciación deportiva que propone el Envigado F.C. es coherente con la estructura que plantea (Renzi, 2009) con unidades de trabajo que propicien el conocimiento, uso y dominio de la propia corporeidad, las habilidades motrices, los juegos motores y las actividades motrices en el medio ambiente, además del estímulo de las capacidades perceptivas, lógico-motrices, relacionales, coordinativas y condicionales, aspectos en los cuales se profundiza de acuerdo al desarrollo filogenético y ontogenético de los infantes, derivando en la optimización del potencial físico, emocional y relacional de cada uno de los participantes del proceso para el mejoramiento de su calidad de vida.

El alcanzar el máximo potencial motriz tiene una relación directa con el mejoramiento de la calidad de vida de los seres humanos, debido a que desde épocas ancestrales hemos sido seres dependientes del movimiento 
para la supervivencia y para la inclusión en la sociedad y aunque los tiempos de la cacería y el nomadismo se han transformado en actividades mucho más sedentarias y dependientes de la tecnología y la automatización, las habilidades del movimiento entregan a los individuos oportunidades para sobresalir en ambientes laborales, deportivos y lúdicos, además de impactar la estructuración de sensaciones y sentimientos positivos que mejoran la autoestima, la seguridad y el autocontrol.

En este caso la investigación se convierte en un elemento que valida la importancia del deporte y la actividad física en la primera infancia como un elemento que aporta a la construcción de una sociedad con individuos capaces, sanos, alegres y conscientes de sus fortalezas, lo que redundará en comunidades competitivas, emprendedoras y cada vez más prosperas.

\section{REFERENCIAS BIBLIOGRÁFICAS}

Ayan P., C. (2013). Valoración de la condición física en el contexto de la educación infantil: aplicaciones prácticas. Apunts. Educación Física y Deportes, 52-62.

Jiménez D., J., \& Araya V., G. A. (2009). Efecto de una intervención motriz en el desarrollo motor, rendimiento académico y creatividad en preescolares. Pensar en Movimiento: Revista de Ciencias del Ejercicio y la Salud, 11-22.

López R., V., \& Castejón O., F. J. (2005). La enseñanza integrada técnico-táctica de los deportes en edad escolar: explicación y bases de un modelo. Apunts: Educación física y deportes, 40-48.

López S., G. F., Borrego B., F. J., \& Díaz S., A. (2013). Efectos de un programa de actividad física en la composición corporal de escolares de 3-5 años. Sportk: revista euroamericana de ciencias del deporte," 41-44.

Oja, L., \& Juerimaee, T. (1997). Assessment of motor ability of 4-and 5-years-old children. American Journal of Human Biology, 559-564.

Orts D., F. (2008). La Iniciación Deportiva: ¿Cuando es el deporte realmente saludable? Actividad Física y Deporte: Ciencia y Profesión, 47-51.

Pérez C., E. A., \& Llames L., R. (2010). Aplicaciones de los Conocimientos Psicológicos en la Iniciación Deportiva. Papeles del Psicólogo, 269-280.

Renzi, G. (2009). Educación Física y Contribución al Desarrollo Integral de los Niños en la Primera Infancia. Revista Iberoamericana de Educación, 50(7), 1-14

Tabernero S., B., Marquez, S., \& Llanos, C. (2002). Elementos a Analizar en el Proceso de Iniciación Deportiva. Retos. Nuevas Tendencias en Educación Física, Deporte y Recreación, 9-15.

Weizmann, F., \& Harris, B. (2012). Arnold Gesell: The Madurationist. En Pickren, W. E., Dewsbury, D. A. \& Wertheimer, M. (Eds) Portraits of pioneers in developmental psychology. New York, NY, US: Psychology Press. 